A. Weisbecker, M. Burmester \& A. Schmidt (Hrsg.): Mensch und Computer 2015 Workshopband, Stuttgart: Oldenbourg Wissenschaftsverlag, 2015, S. 589-594.

\title{
Das Projekt PAnalytics - Selbstmonitoring für gesundes Altern
}

\author{
Nils Beckmann, Aysegül Dogangün, Katja Herrmanny, Hanno Sauer, Katha- \\ rina Kloppenborg
}

Kompetenzzentrum Personal Analytics, Universität Duisburg-Essen

\section{Zusammenfassung}

Die stetige Weiterentwicklung und Vernetzung von Personal-Health-Geräten, tragbaren und ambienten Sensoren und Smartphones ermöglicht die immer genauere Messung und Sammlung gesundheitsrelevanter Daten. Bedingt durch den demographischen Wandel gewinnen neue Konzepte für ein gesünderes Leben immer mehr an Relevanz. Während es bereits viele sogenannte „Insellösungen“ gibt, die bestimmte gesundheitsrelevante Parameter erfassen und auswerten, mangelt es an einem Ansatz im Gesundheitsmonitoring, der auf Basis einer Vielzahl gesundheitsrelevanter Daten Handlungsempfehlungen generiert und von der Zielgruppe „,50Plus“ selbstständig nutzbar ist. Das Projekt PAnalytics will sich in einer interdisziplinären Forschungsgruppe diesem Problem widmen und ein ganzheitliches personalisiertes Selbstmonitoring-System entwickeln. Ziel dieses Beitrags ist es, die Ansätze des Projektes vorzustellen, sowie die Notwendigkeit der interdisziplinären Zusammenarbeit aufzuzeigen.

\section{Einleitung}

Der demographische Wandel besteht in der beständigen Alterung der Gesellschaft. Neue Konzepte für ein gesünderes Leben, für den längeren Erhalt in der Arbeitswelt und für ein effizienteres sowie kostengünstigeres Gesundheitssystem mit fitteren älteren Bürgern sind somit unumgänglich (Baltzer \& Mertens, 2014). Individualität und Lebensqualität gewinnen immer mehr an Relevanz und liegen primär in individueller Verantwortung (Schmidt, 2008).

Es gibt bereits eine Vielzahl von Menschen, die ein bewusstes gesundheitsorientiertes Leben führen, indem sie ihre Gesundheit und ihr Wohlbefinden umfassend auswerten (Choe et al., 2014). So gehört das regelmäßige Messen und Dokumentieren der Vitalparameter und Aktivitäten für chronisch erkrankte Menschen oder Sportler zum Alltag. Die Vernetzung von Personal-Health-Geräten wie Waagen, Blutdruckmessgeräten oder Schrittzählern und die weite 
Verbreitung von Smartphones und entsprechenden Apps haben diese Form des Selbst-Monitoring in den letzten Jahren begünstigt (Swan 2012) und sind auch für eine ältere Nutzergruppe relevant (Mercer et al. 2015). Der korrekte Umgang mit einer Vielzahl von auf einen Bereich spezialisierten Einzelkomponenten, auch „Insellösungen“ genannt, sowie die Notwendigkeit der selbstständigen Interpretation der Bedeutung der Daten für die eigene Gesundheit erfordern jedoch ein technisches sowie gesundheitswissenschaftliches Know-How (Choe et al., 2014). Das Projekt PAnalytics, kurz für Personal Analytics, hat daher das Ziel, ein personalisiertes System für die Lebensumgebung zu entwickeln, das den Erhalt der Gesundheit und eines „selbstständigen“ Lebens zuhause mittels eines ganzheitlichen gesundheitsbezogenen Monitoring durch den Nutzer selbst erlaubt.

\section{Stand der Technik im Gesundheitsmonitoring}

Das regelmäßige Messen und Dokumentieren von Körperwerten im Alltag zur gesundheitsbezogenen Selbstanalyse ist in Forschung und Wirtschaft als Trend zu verzeichnen. Insbesondere Anwendungen zur Nutzung von Smartphones mit ihren integrierten Sensoren als „Gesundheitshandy“, sowie tragbare Sensorik im Sinne des wearable computing werden immer beliebter (Steinhubl et al. 2015). Derzeitig einschlägige Forschungs- und Entwicklungsaktivitäten im Bereich Personal Analytics richten sich primär auf die Entwicklung von „Insellösungen“. So gibt es Tools, die beispielsweise Glukosewerte ${ }^{1}$, den Urin $^{2}$ oder das Herz ${ }^{3}$ überwachen. Smartphone-Applikationen unterstützen den Nutzer z.B. beim Atmen ${ }^{4}$ oder untersuchen Stimmung und Lebensqualität ${ }^{5}$. Auf einer höheren Ebene nutzen z. B. Open.Sen.se ${ }^{6}$ und Tictrac $^{7}$ die Daten aus verschiedenen sozialen Onlineplattformen, Handyapplikationen und Trackern und bewerten und belohnen die Aktivitäten des Anwenders. Die meisten Forschungsarbeiten und Produkte konzentrieren sich also auf einen speziellen Anwendungsbereich und lassen einen ganzheitlichen Betrachtungsaspekt aus. Die letztgenannten, höheren Anwendungen bündeln zwar eine Vielzahl an Sensoren und ermöglichen die grafische Auswertung der Daten, überlassen jedoch die Interpretation der Informationen dem Benutzer. Die meisten PersonalAnalytics-Systeme für ältere Menschen beruhen auf einer gesundheitlichen Überwachung der Person mit einer Art Frühwarnsystem für Angehörige und Betreuungspersonen (Baltzer \&

1 Dexcom, http://www.dexcom.com/dexcom-g4-platinum-share, 2012, zuletzt gesehen am 19.05.2015

2 Piddle, http://www.linehq.com/showcase/piddle, 2013, zuletzt gesehen am 19.05.2015

3 Alivevor, http://www.alivecor.com, 2011-2014, zuletzt gesehen am 19.05.2015

4 Williams, S. W., Townsend, M., Breathe Syncm. http://www.breathesync.com, 2013, zuletzt gesehen am 19.05.2015

5 HeartMath, http://www.heartmathstore.com/item/6400/inner-balance, 2014, zuletzt gesehen am 19.05.2015

6 Open.Sen.se., von http://open.sen.se, 2014, zuletzt gesehen am 19.05.2015

7 Tictrac, https://www.tictrac.com, 2010-2014, zuletzt gesehen am 19.05.2015 
Mertens, 2014) ${ }^{8}$. Aktuelle wissenschaftliche Arbeiten zeigen, dass derzeitige Gesundheitsmonitoring-Tools nicht ausreichend verständlich und auf die Selbstreflexionsbedürfnisse der Nutzer abgestimmt sind (Li et al. 2011; Oh \& Lee, 2015) und dass zur weiteren Forschung an persuasiven Systemen die Durchführung von klinischen Studien, wirtschaftliche und soziale Analysen sowie Nutzerstudien notwendig sind (Orwat et al. 2008).

Es sind bereits einige ganzheitliche Ansätze in der Forschungslandschaft vorhanden. In Lee und Balan (2014) wird ein Personal Analytics Layer vorgestellt, das Nutzerdaten aus verschiedenen Sensor-, Telefon- und Online-Daten nutzt, um zum Beispiel Emotionen und Vorlieben von Nutzern abzuleiten. Tollmar et al. (2012) präsentieren mit „Mobile Health Mashups“ die kombinierte Auswertung gesundheitsrelevanter Daten aus verschiedenen Apps. Die Forschung an ganzheitlichen, selbstständig zu nutzenden Gesundheitsmonitoringsystemen ist generell noch sehr jung und bedarf weiterer Arbeiten. Selbst zu nutzende Ansätze in der häuslichen Umgebung sind aufgrund des demographischen Wandels besonders für ältere Menschen relevant, damit diese möglichst lange gesund bleiben und in ihrer häuslichen Umgebung unterstützt leben können.

\section{PAnalytics - ein interdisziplinärer Ansatz}

Den bisherigen Lösungen im Bereich des Gesundheitsmonitoring fehlt ein ganzheitlicher Ansatz, der Daten aus unterschiedlichen Geräten fusioniert mit weiteren multimodal und gegebenenfalls implizit erfassten Alltagsaktivitätsdaten aus der Wohn- oder Lebensumgebung sowie expliziten Nutzereingaben und Daten aus sozialen Netzwerken kombiniert, auswertet und darstellt. Im Rahmen des Projektes PAnalytics soll dieser ganzheitliche Ansatz verfolgt werden. Das Ziel des Projektes ist die Entwicklung eines umfassenden, integrierten SelbstmonitoringSystems, das in der Erhebung von Gesundheitsparametern, der Erkennung von gesundheitsrelevanten Verhaltensmustern, der Ableitung von Empfehlungen, deren persuasiver Darstellung innovativ und im Hinblick auf ethische und datenschutzrelevante Aspekte vorbildlich ist. Das System umfasst ein Monitoring und die gesundheitswissenschaftliche Auswertung von Ernährung, Bewegung, sozialer Interaktion, seelischem Befinden und Schlafqualität. Die Interpretation der erfassten und visualisierten Daten soll nicht mehr dem Nutzer überlassen werden, sondern ein System mit gesammeltem Expertenwissen soll die gesundheitsbezogenen Daten interpretieren und Vorschläge für gesundheitsfördernde Aktivitäten und Verhaltensweisen zur Verbesserung der Lebenssituation anbieten. Eine zielgruppenspezifische Ergänzung um medizinische Monitoring- und Assistenzfunktionalitäten soll den Bedürfnissen des Anwenderkreises ,50Plus“" gerecht werden.

Eine interdisziplinäre Aufstellung des Projektes mit den Disziplinen Elektrotechnik, Informatik, Kognitionswissenschaft, Gesundheitswissenschaft und Ethik ermöglicht es, die oben genannten bestehenden Lücken von in der Vergangenheit erfolgten Ansätzen im Bereich des Gesundheitsmonitorings zu schließen und eine ganzheitliche Lösung zu entwickeln.

8 Dool, T., QS - Livind, http://quantifiedself.com/elderly/, 2011, zuletzt gesehen am 19.05.2015 


\subsection{Elektrotechnik}

Im Projekt ist als wesentlicher Fortschritt gegenüber existierenden Ansätzen geplant, die Informationsdarreichung an den Nutzer von dessen Situation und Befinden abhängig zu machen und so die Mensch-Maschine-Interaktion zu verbessern. Ein besonderer Fokus im Projekt liegt daher darauf das seelische Befinden (synonym für Wohlgefühl, Balance, Ausgeglichenheit, Emotion, Stress) „,messbar“ zu machen. Die Entwicklung aus Sicht der Elektrotechnik konzentriert sich auf Techniken die raumgestützt oder (z.B. textilintegriert) mobil funktionieren und für die Nutzer mit keinerlei Einschränkungen in ihrer Bewegungsfreiheit und für ihr Handeln verbunden sind. In Frage kommen Haltungs-, Gestik- und Mimik-Erkennung, die Stimmanalyse (Schuller et al. 2014), die Gesichtsthermographie (Janssen et al. 2012) und die Ableitung physiologischer Parameter (Kreibig 2010).

\subsection{Informatik}

Die Herausforderungen der Informatik bestehen in der Herleitung neuer Formen der Aktivitätserkennung in häuslichen Umgebungen und im Lebensumfeld, sowie der Aufstellung von Methoden zur Big-Data-Analyse. Alltägliche Routinen sollen aus einer Reihe von Beobachtungen wie Handlungen und Umweltbedingungen erkannt werden (Choudhury et al. 2008; Munstermann 2015). Kausale gesundheitsrelevante Zusammenhänge sollen maschinenverständlich abgebildet werden, um über eine Wissensbasis aus Beziehungen zwischen Alltagsaktivitäten wie Bewegung, Schlaf und Ernährung gesundheitsrelevante Empfehlungen generieren zu können. Zu diesem Zweck müssen außerdem Metriken entwickelt werden, die gesundheitsrelevante Aktivitätscluster wie beispielsweise Schlaf, soziale Interaktion oder Befinden formalisieren, z. B. in Form stochastischer Modelle (Dogangün 2012), und somit bewertbar machen.

\subsection{Kognitionswissenschaft}

Die Aufgabe der Kognitionswissenschaft ist es, die vom System abgeleiteten Empfehlungen in motivierender Form darzustellen, um die Nutzer bei der angestrebten Verhaltensänderung zu unterstützen. Zur Realisierung dieses Anliegens werden gezielt mit empirischen Untersuchungen motivationale und persuasive Aspekte von Gesundheitsmonitoringsystemen erforscht und die entsprechenden Systemkomponenten in einem nutzerzentrierten Vorgehen entwickelt. Ein Betrachtungsfokus wird dabei die individuelle Anpassung der Empfehlungen und Feedbacks auf Basis des aktuellen Wohlbefindens der älteren Personen und unter Berücksichtigung weiterer Kontexteinflüsse sein. Das Ziel ist es, langfristige Verhaltensänderungen im Sinne einer aktiven Gesundheitsförderung zu erzielen, die ein gesundes Altern unterstützen.

\subsection{Gesundheitswissenschaft}

Die gesundheitswissenschaftliche Begleitung der Nutzerstudien soll mehr Erkenntnisse liefern, als bisher in der Forschung zu persuasiven Systemen vorhanden ist. Mit einer bisher nicht 
dagewesenen Form der Datenaufnahme und Quantifizierung der Lebensweise werden Zusammenhänge zwischen dem Lebensstil und der eigenen Gesundheit bzw. Krankheit erarbeitet und dabei inter-, sowie intraindividuelle Unterschiede berïcksichtigt. Das Ziel ist die Sammlung und Aufbereitung von Expertenwissen, und zwar derart, dass um den Nutzer herum gesammelte Daten gesundheitsbezogen interpretiert und Vorschläge für gesundheitsfördernde Aktivitäten und Verhaltensweisen zur Verbesserung der Lebenssituation angeboten werden können.

\subsection{Ethik}

Die Beteiligung der Ethik bildet einen großen Mehrwert, indem ethisch-soziale Auswirkungen, Datenschutz- und -sicherheitsaspekte, die bisher vernachlässigt wurden, bereits in der Konzeptionierungsphase berücksichtigt werden können. Das System muss der Realität pluralistischer Gesellschaften gerecht werden und vermeiden, ein einziges Modell des gesunden Lebens durchzusetzen und damit eine Art technologischen Paternalismus zu etablieren. Allein die Tatsache, dass die Gesundheitsüberwachung normativ nicht neutral ist in Bezug auf moralische gehaltvolle Konzepte wie Gesundheit, Krankheit, Normalität und Alter, ist jedoch kein generelles Argument gegen Monitoringsysteme. Es ist allerdings ein Argument für eine sorgfältige ethische und philosophische Analyse dieser Konzepte und deren Umsetzung in der Entwicklung, die über den gesamten Projektverlauf erfolgen wird.

\section{$4 \quad$ Fazit}

Die Sichtung der aktuellen Forschungslandschaft und des Stands der Technik zeigen, dass kein ganzheitlicher von der Zielgruppe ,50Plus“ selbst zu nutzender Ansatz des Gesundheitsmonitoring existiert, wie er im Rahmen des Projekts angestrebt wird. Durch interdisziplinäre $\mathrm{Zu}-$ sammenarbeit will PAnalytics diese Lücke schließen und Anwendungssysteme, die den oben genannten Ansatz verfolgen, entwickeln.

\section{Literaturverzeichnis}

Alkhatib, A., \& Boellstorff, T. (2014). Quantified Self: Ethnography of a Digital Culture (Doctoral dissertation, Doctoral dissertation, University of California). Retrieved from http://alialkhatib. com/presentations/QSThesisFinal. pdF).

Baltzer, D. W. I. M., \& Mertens, A. (2014). Technische Unterstützung für gesellschaftsfähiges Altern. Zeitschrift für Epileptologie, 27(4), 276-278.

Choe, E. K., Lee, N. B., Lee, B., Pratt, W., \& Kientz, J. A. (2014, April). Understanding quantifiedselfers' practices in collecting and exploring personal data. In Proceedings of the 32nd annual ACM conference on Human factors in computing systems (pp. 1143-1152). ACM.

Choudhury, T., Consolvo, S., Harrison, B., Hightower, J., LaMarca, A., LeGrand, L., ... \& Haehnel, D. (2008). The mobile sensing platform: An embedded activity recognition system. Pervasive Computing, IEEE, 7(2), 32-41. 
Doğangün, A. (2012). Adaptive Awareness-Assistenten: Entwicklung und empirische Untersuchung der Wirksamkeit (Vol. 32). BoD-Books on Demand.

Janssen, J. H., van den Broek, E. L., \& Westerink, J. H. (2012). Tune in to your emotions: a robust personalized affective music player. User Modeling and User-Adapted Interaction, 22(3), 255-279.

Kreibig, Sylvia D.:Autonomic nervous system activity in emotion: a review. In: Biological psychology 84 (3) ,2010, S. 394-421. DOI: 10.1016/j.biopsycho.2010.03.010.

Lee, Y., \& Balan, R. K. (2014, June). The case for human-centric personal analytics. In Proceedings of the 2014 workshop on physical analytics (pp. 25-29). ACM.

Li, I., Dey, AK. \& Forlizzi, J. (2011). Understanding my data, myself: supporting self-reflection with ubicomp technologies. In Proceedings of the $13^{\text {th }}$ international conference on Ubiquitous computing, New York: ACM, S. 405-414.

Kreibig, S. D. (2010). Autonomic nervous system activity in emotion: A review. Biological psychology, 84(3), 394-421.

Mercer, K., Baskerville, N., Burns, C. M., Chang, F., Giangregorio, L., Goodwin, J. T., ... \& Grindrod, K. (2015). Using a Collaborative Research Approach to Develop an Interdisciplinary Research Agenda for the Study of Mobile Health Interventions for Older Adults. JMIR mHealth and uHealth, $3(1)$.

Munstermann, M. (2015). Ambiente Assistenzfunktionen. In Technisch unterstützte Pflege von morgen (pp. 41-102). Springer Fachmedien Wiesbaden.

Oh, J., \& Lee, U. (2015, January). Exploring UX issues in Quantified Self technologies. In Mobile Computing and Ubiquitous Networking (ICMU), 2015 Eighth International Conference on (pp. 53-59). IEEE.

Orwat, C., Graefe, A., \& Faulwasser, T. (2008). Towards pervasive computing in health care-A literature review. BMC Medical Informatics and Decision Making, 8(1), 26.

Schmidt, B. (2008). Eigenverantwortung haben immer die Anderen. Der Verantwortungsdiskurs im Gesundheitswesen. Bern: Huber.

Schuller, B., Steidl, S., Batliner, A., Schiel, F., \& Krajewski, J. (2014). Introduction to the Special Issue on Broadening the View on Speaker Analysis. Computer Speech and Language, 28(2), 343-345.

Steinhubl, S. R., Muse, E. D., \& Topol, E. J. (2015). The emerging field of mobile health. Science translational medicine, 7(283), 283rv3-283rv3.

Swan, M. (2012). Sensor mania! the internet of things, wearable computing, objective metrics, and the quantified self 2.0. Journal of Sensor and Actuator Networks, 1(3), 217-253.

Tollmar, K., Bentley, F., \& Viedma, C. (2012, May). Mobile Health Mashups: Making sense of multiple streams of wellbeing and contextual data for presentation on a mobile device. In Pervasive Computing Technologies for Healthcare (PervasiveHealth), 2012 6th International Conference on (pp. 6572). IEEE.

\section{Kontaktinformationen}

Kompetenzzentrum Personal Analytics

Gruppenleitung Dr.-Ing. A. Dogangün, ayseguel.doganguen@uni-due.de 